\author{
Geoffrey Goldspink ${ }^{\mathrm{a}}$ \\ Kay D. MacDermot ${ }^{\mathrm{b}}$ \\ Francisco J. Novo ${ }^{\mathrm{c}}$ \\ a Department of Anatomy and \\ Developmental Biology, \\ b The Clinical Genetics Unit, \\ The Royal Free Hospital School of \\ Medicine, London, UK; \\ c Department of Genetics, \\ University of Navarra, Spain
}

\section{Key Words}

Gene therapy

Genomic profile

Cloning humans

Abuse of new methods

Tissue engineering

\title{
Social and Moral Implications Arising from Genetic Screening and Genetic Engineering
}

\begin{abstract}
The development of techniques for altering the genetic makeup of humans as well as animals has proceeded at a much faster pace than the discussion of the moral and social implications. The human genome project aims at sequencing all the genes that are required to form a human being. The use of genetic profiles that appear to deviate from the ideal for assessing individuals for jobs or insurance cover is an aspect of this new science that will probably continue to present ethical problems. There are unquestionable benefits from treating hitherto incurable diseases using gene therapy and also from being able to produce pharmacologically active proteins in bacteria. Examples are given of how these are open to abuse. The development of techniques for cloning mammals including man also provides a moral dilemma as to where to draw the line between cloning human cells to reconstruct diseased organs and cloning a whole person. Therefore a total ban world-wide on cloning human tissue is perhaps impracticable and undesirable.
\end{abstract}

\section{Introduction}

The most rapidly expanding area in biomedical science is that of molecular genetics. Although it results in enormous benefits for mankind, it presents ethical problems that were, hitherto, unthinkable. This is high- lighted by the recently reported cloning of a mammal using a technique that could be in principle readily extended to cloning human beings. In this article we attempt to highlight the background and ethical dilemmas that stem from genetic engineering. It is not an attempt to form judgement with regard to any

\begin{tabular}{ll}
\hline KARGER & $\begin{array}{l}\text { (1) 1998 S. Karger AG, Basel } \\
\text { 1011-7571/98/0072-0150\$15.00/0 }\end{array}$ \\
$\begin{array}{l}\text { Fax +4161306 12 34 } \\
\text { E-Mail karger@karger.ch } \\
\text { www.karger.com }\end{array}$ & $\begin{array}{l}\text { This article is also accessible online at: } \\
\text { http://BioMedNet.com/karger }\end{array}$
\end{tabular}

Prof. Geoffrey Goldspink

Division of Basic Medical Sciences, Royal Free Hospital School of Medicine, University of London, Rowland Hill Street

London NW 32 PF (UK)

Tel. +44 0171794 0500, ext. 4355, Fax +44 01718302917 
of the procedures relating to manipulation of reproduction and the use of gene transfer methods.

\section{Background}

\section{Proteins and Genes}

The structural material of life, the enzymes and many of the hormones that regulate our bodily functions are proteins encoded in a chemical substance called deoxyribonucleic acid (DNA). This substance contains our genetic information and together with proteins makes up each of our 46 chromosomes. Occasionally, mutations occur, some of which may have disastrous consequences. A person has about 100,000 genes received from each parent, so each person has a double set of genes contained in a double set of chromosomes. The mechanism involves the recombination or reshuffling of the genetic material and this results in a 'different genetic mix' each time. In some genes, the maternal gene is expressed and in other genes the paternal gene is expressed. Thus each newborn child is a unique individual with a unique potential and not an exact replica of its father and mother. Hence two parents of moderate ability may produce a child with very high or low intelligence. Obviously, the genetic make-up is not the only factor that will determine how a child will develop. This involves 'nurture and nature' which is another way of saying that the environment in which the child is raised determines whether the child will achieve his/her full potential.

\section{The Genetic Code}

The genetic code is a beautifully simple method of coding vast amounts of hereditary information in a very small volume of DNA. This information is replicated billions of times as the single fertilized ovum (egg) divides and divides to produce the billions of cells that make up the body. When the hereditary information is replicated each time, each cell receives the complete message or complete 'set of blueprints'. This is done virtually without error because there is a proof-reading system which checks to see that none of the 3,000 million nucleotide bases or letters that make up this information finishes up in the wrong order. Errors which do occur (mutation) may be of no consequence, or may cause or predispose to disease.

Each gene codes for one or more different proteins and this is done arranging the 20 different amino acids in various orders. DNA is made up of four different bases or letters and different combinations of three of each of these four letters code for a different amino acid. Three thousand million bases are necessary to encode the information necessary for a human being to develop. These are in the form of sentences or genes in which the bases are arranged in triplets of three-letter words, plus stop and start signals. Just one base in the incorrect order may result in such devastating conditions as muscular dystrophy, sickle cell anaemia, or cystic fibrosis. At the present time the Human Genome Project aims to map the whole of the human genome, in other words, determine the order of the 100,000 genes and the sequence of the 3,000 million bases which code the genetic information. This is the multi-million dollar project of the same magnitude as the cost of landing a man on the moon. The question is, what will this information be used for and should there be restrictions on the application of the knowledge.

\section{Gene Engineering}

The term genetic engineering in some people's minds conjures up the image of animals or humans with two heads or grotesque fea- 
tures. But engineered human genes have already been introduced into bacteria, which produce human insulin for the treatment of diabetes or human growth hormone for the treatment of pituitary dwarfism. Sometimes bacteria cannot express the human genes in the correct way, so another strategy has been used. Human genes were inserted into developing sheep or goat embryos, so that the adult animal was able to express human protein in the milk. Therapeutic substances produced in this way include: tissue plasminogen activator to treat thrombosis, alpha-1-antitrypsin for the treatment of emphysema caused by this enzyme deficiency and clotting factor IX which is lacking in haemophilia.

Genes from other species can be inserted into embryos of farm animals to improve their disease resistance and their food and fibre value. Is this creating new forms of life and is it any different to the selective breeding of livestock or pet animals that has been carried out for centuries? The moral issues include questions such as, should genes of any species including human genes be put into other animals? The human growth hormone gene has been inserted into pigs' genome in an attempt to make pigs grow faster. If these pigs are eaten, does this constitute cannibalism? Strong popular reaction against genetically enhanced food has developed in several Western countries, and debate about genetic engineering will no doubt increase in the near future.

\section{Genetic Screening}

Information provided by molecular genetics is already being employed in paternity testing and in forensic medicine. A method called genetic fingerprinting is used which is based on the detection of spacing sequences in DNA called minisatellites or microsatellites which are not genes but parts of the DNA that show a lot of variation between individuals. Genetic variability in these regions is of no consequence as the regions do not code for a specific protein. Mini- and microsatellites are similar between parents and offspring, but different between unrelated individuals. Analysis can be performed on a drop of blood or merely a flake of skin. A recent attempt by the US military to create a database containing this type of information about Army recruits has faced strong opposition by the general public and has been challenged in Court. It is perceived by the public that adequate control of such a vast amount of genetic information about individuals could be easily misused.

The progress of the Human Genome Project has led to the identification of many genes responsible for hereditary disorders. This opens the possibility of detecting healthy carriers of serious genetic disorders before they begin reproductive life. As efforts are being aimed at the identification of genes involved in susceptibility to common polygenic disorders, such as hypertension and coronary heart disease, there is a strong concern about whether insurance companies or prospective employers will have access to this information, and whether they will insist on individuals subjecting themselves to genetic testing before they can be insured, given a mortgage or offered a job. This will create a 'genetic underclass' (Verity 1997) who will be unable to buy a house or obtain a job. They may find it difficult to register with a family doctor in a system such as operated in the UK based on fund holding, or to join the medical insurance system in other countries, as these patients will inevitably incur higher costs for the practice. With the insurance system even if they obtain employment, it would be difficult to change jobs as these individuals would have difficulty in obtaining a new medical insurance. 


\section{In vitro Fertilization, Pre-Implantation Diagnosis and Abortion}

The moral issues raised by in vitro fertilization (IVF) have been well publicized in newspapers, as was surrogate motherhood, when the source of the sperm or the egg is from an individual other than the marriage partner. By manipulating hormone levels and preserving eggs and sperm by deep freezing, IVF techniques have unquestionably helped many married couples to realize their desire to have children, but this also poses important ethical questions. The artificial insemination of single unmarried women has been opposed by the major Christian denominations and the Muslim and Jewish religions on the grounds that marriage is the natural state ordained by God for the procreation of children. IVF procedures have also been used in women who are well beyond the normal childbearing age (60 years) and this has raised the question about child welfare, being cared for by parents at the end of their natural life span.

Before the IVF procedure, superovulation is induced in the female by hormonal injections, which results in the release of the ovary with 1-12 oocytes (unfertilized eggs). After fertilization in the 'test tube' with their father's sperms only 3 of these fertilized oocytes are implanted into the mother to prevent multiple pregnancy with poor prognosis for survival. The other fertilized oocytes, which are at the stage of 4- to 8-cell embryos, are frozen as the procedure often has to be repeated. The Human Fertility and Embryology Authority in the UK set a 5-year limit on storage of the remaining embryos. The moral issues raised by this procedure have recently come to the fore when the first 5-year deadline expired and many of these embryos were destroyed, as the prospective parents did not come forward. Does this constitute destroying human life?
As an extension of the IVF procedures, it is now possible to screen the early embryos for a range of hereditary diseases and for gender. A few cells can be removed and the DNA of these cells is amplified and analysed so that the early embryo free of genetic defect can be implanted back into the mother or into a surrogate mother. The moral issue here is to what extent these powerful molecular genetic methods should be used for the selection of embryos that will be allowed to develop. In other words, could this new knowledge be used to determine the characteristics (sex, physical stature, intelligence, life expectancy) of our future offspring? There seems to be a strong case for testing early embryos when there is a family history of serious hereditary diseases such as Duchenne muscular dystrophy, in other words, when it is clinically indicated. It is frequently claimed that pre-implantation diagnosis may reduce the number of therapeutic abortions, if the early embryo with serious medical defects can be detected. It can be envisaged that IVF will become a more common place as the methods for IVF become more successful. We must therefore anticipate the moral challenges arising if these methods are to be used to select for certain characteristics such as height or sex of the child, rather than serious medical disorders.

The techniques of amniocentesis and fetoscopy allow small tissue samples to be obtained from the fetus in utero. These samples can be screened for many genetic defects. In cases where there is a family history of a certain hereditary disease and the offspring has inherited the defective gene, the parents are given the option of proceeding with an abortion. It should be pointed out that this is not a way to eradicate hereditary diseases completely as these often result from spontaneous mutations and can occur in any family at any time. For example, 1 in 3 cases of Duchenne muscular dystrophy arises from a new mutation. 
Even if all pregnant women were screened and the affected fetuses aborted, this would still not eradicate such common disorders as Duchenne muscular dystrophy. The way forward is to treat these conditions soon after birth, and gene therapy methods employing engineered genes to correct the genetic defect are being investigated (see below).

The big issue underlying the moral questions posed by abortion and pre-implantation diagnosis is the status of the early human embryo: is it a human person or just a clump of cells? The major religions and most agnostics believe in the sanctity of human life. From a genetic standpoint it is clear that a new genetic entity is produced after the fusion of the pronuclei at the end of the fertilization of the egg by the sperm cell. Genome activation takes place at the 4-cell embryo stage, and epigenetic mechanisms of gene regulation such as imprinting and inactivation of the chromosome in females are already operative at least in the 8-cell embryo, a point at which irreversible commitment to human development exists. However, there is some controversy concerning the beginning of human moral status, and it has been suggested that a developing human life would not necessarily be a human person deserving the same degree of respect as a more developed form. For instance, it is sometimes claimed that the human person arises gradually as the human embryo develops [1], but this could also be applied to adult life until the very moment of death. More frequently it has been suggested that the human person begins when the developing embryo acquires a certain set of characteristics or functions. The problem with this approach is defining the collection of characteristics needed to grant human moral status. These definitions will vary greatly in different cultures and even between different authors, making consensus in this important issue very unlikely. Finally, another common opinion is that the early developing human shows the full active potential to become a mature human person and should be given full human status [3]. Although this fits in better with the biology of early human development, it is in direct conflict with positions that justify the implementation of abortion in certain cases or the selective destruction of embryos during IVF procedures. Obviously, further debate in this difficult area is needed, as it is probably the crucial ethical issue confronting those involved in these areas.

\section{Gene Therapy}

There are at least 4,000 genetic diseases known at present and the number increases as we learn more about the traits our genes determine. Common conditions, such as coronary heart disease, Alzheimer's disease, dementia, cancer and progression of AIDS can be largely genetically determined. The prospects for treatment for these diseases lie in the identification and then modification of these genes by gene therapy methods. For example, Duchenne muscular dystrophy is due to the affected muscles' inability to produce the protein dystrophin. This is coded for by one of the largest genes known (2.5 million bases) and one or a few bases missing or in an incorrect position results in the absence of the protein or in a defective protein being produced. This protein is required to stabilize the membrane of the muscle fibres and in its absence the cells degenerate. One possibly curative treatment for this serious condition which affects 1 in 3,000 males is to introduce the normal dystrophin gene into as many muscle fibres as possible. The possibilities now exist for doing this by using a disabled virus which has been engineered to include the dystrophin gene. Immune deficiency diseases may be treated by gene replacement. The patient's 
bone marrow cells are grown in culture, and the appropriate genes can be introduced into the stem cells. These can be injected into the blood stream to repopulate the bone marrow, and produce corrected lymphocytes. None of these attempts at treatment have produced a 'cure' to date, but these techniques are under intensive research world-wide.

The main ethical issues raised by gene therapy strategies are mainly those concerned with the safety of the procedures. This is specially relevant when viruses are used as vectors to carry the normal gene, as they theoretically could in the long term result in malignancies or in diseases related to the host reaction against the vectors or against the transgene. Gene therapy is a rapidly growing field which is still in its infancy but the first clinical applications are now on the horizon e.g. cystic fibrosis. The possibility exists therefore for developing potential cures for diseases that still plague mankind. This, in turn, will produce many of the ethical problems mentioned above, as new therapeutic alternatives will allow embryos with inherited defects to be born with the prospect of disease-free lives. However, these techniques will in principle also allow the introduction of any other genes not associated with human disease. It would be possible for example to enhance muscle growth by introducing genes which result in the overexpression of growth hormone and other factors and thus build a super athlete but no doubt with some side-effects. The acceptability by the public of this application will have to be determined.

\section{Cloning and Manipulation of the Genome of Animals and Human Beings}

The prospects of cloning humans have again been highlighted by the recent reports of the successful cloning of a sheep at a Scottish

Social and Moral Implications Arising

from Genetic Screening and Genetic

Engineering
Agricultural Institute using a technique that involved taking the nucleus from a single cell from the udder of a mature sheep and inserting it into an enucleated donor egg [4]. This resulted in an animal that was genetically identical to the donor animal. Other species had previously been cloned this way, e.g. the Xenopus toad, but they did not survive through to adulthood. The fact that the cloning technique has been successfully used on mammals means that it could be applied to human beings. At the present time regulations regarding cloning of humans are being hastily produced, but it is unlikely that a world-wide ban could be enforced. It should be pointed out, however, that there are potential problems using this cloning method as the chromosomal material in somatic cells shows signs of ageing in that the length of telomeres decreases. Therefore the offspring might be expected to show premature ageing. Other cloning or transgenic biology research has been in the headlines. For instance it has been possible to produce pigs in which the tissues have been humanized. The purpose of this work is to produce genetically altered pigs so that their organs can be used for human transplantation. The transplantation of animal tissues into human patients results in a strong acute immunological rejection as the tissue is recognized as foreign. Humanization of the tissues means that hearts, livers and kidneys could be used for transplantation with a high degree of success. At the present time this is achieved by injecting multiple copies of an engineered gene into the recently fertilized ovum. Another approach that has been perfected in the cloning of mice is the use of embryonic stem cells. These are transfected with the gene in question and then introduced into the blastocyst. This opens up the possibility for cloning as well as gene manipulation.

The go-ahead for transplanting these genetically modified spare organs has been de- 
layed until the possibility of transferring viruses from the pigs to man has been fully investigated. The extreme scenario is to clone human 'spare parts' in human fetuses rather than pigs. However as tissue engineering techniques are developed there is the possibility of cloning genetically altered human cells and even allowing these to be formed into any embryonic organ in culture or in animals. A human external ear has been formed on the skin of a mouse. This was used for surgical transplantation onto a child who had a con- genitally malformed right ear. Gene manipulation and cellular cloning may be acceptable for the purposes of making spare parts in vitro. However would it be acceptable to clone a whole human being from say a recently dead child when requested by a distraught mother? This poses the question, how far can genetic engineering and cloning be allowed to develop in order to improve health care without undermining the basic tenet of the sanctity of human life?

\section{References}

1 Godfrey J: The Pope and the ontogeny of persons. Nature 1995;373: 100.

2 Opinion: Human cloning requires a moratorium, not a ban. Nature 1997;386:1.
3 Watt H: Potential and the early human. J Med Ethics 1996;22:222.

4 Wilmut I, et al: Viable offspring derived from fetal and adult mammalian cells. Nature 1997;385:810813. 